\title{
A NOTE ON THE SINGULARITIES IN THE THEORY OF WATER WAVES WITH AN INERTIAL SURFACE
}

\author{
B. N. MANDAL AND KRISHNA KUNDU ${ }^{1}$
}

(Received 3 June 1985; revised 11 October 1985 and 24 January 1986)

\begin{abstract}
This note is concerned with the derivation of velocity potentials describing the generation of infinitesimal gravity waves in a motionless liquid with an inertial surface composed of uniformly distributed floatıng particles, due to fundamental line and point sources with time-dependent strengths submerged in a liquid of finte constant depth.
\end{abstract}

\section{Introduction}

Velocity potentials due to fundamental time-harmonic singularities present in an ideal liquid with a free surface are useful in studying the problems of scattering or radiation of surface waves involving obstacles either partially immersed or fully submerged in the liquid. Thorne [5] listed the different forms of velocity potentials due to different kinds of time-harmonic singularities present in the liquid with a free surface and Rhodes-Robinson [2] modified these results by taking into account the effect of surface tension of the free surface. These time-harmonic potential functions are in fact solutions of certain boundary-value problems satisfying the Laplace's equation in the liquid medium except at a point where they are singular in a prescribed manner and satisfy a Churchill type boundary condition at the free surface.

Instead of a liquid with a free surface, if we have a liquid covered by an intertial surface composed of a thin uniformly distributed floating matter (e.g. broken ice), propagation of time-harmonic progressive gravity waves of any

\footnotetext{
${ }^{1}$ Department of Applied Mathematics, University of Calcutta, 92, A.P.C. Road, Calcutta-700009, India.

(ㄷ) Copyright Australian Mathematical Society 1986, Serial-fee code 0334-2700/86
} 
angular frequency is not possible if the inertial surface is too heavy (cf. RhodesRobinson [3]). This necessitates considering initial value problems involving different types of singularities with time-dependent strengths. Rhodes-Robinson [3] considered a two-dimensional wave source submerged in a liquid of infinite depth with an inertial surface. In the present note we extend this to the case of finite depth of liquid including other different types of singularities from which infinite depth results can be obtained as a limiting case. We consider in $\$ 3$ a variable two-dimensional wave source present in a liquid of finite depth, and in $\$ 4$ variable point singularities (three-dimensional multipoles) also in liquid of finite depth. It is believed that the potential functions derived here and in [3] due to different types of singularities will be of use when problems associated with obstacles of different geometrical shapes present in a liquid with an inertial surface are considered.

\section{Statement of the problem}

We choose a rectangular cartesian coordinate system where the $y$-axis is taken vertically downwards and $x z$ plane is horizontal. Let an ideal liquid occupy the region $0<y<h, h$ being the constant depth of the liquid, $\rho$ being its volume density, and its surface being completely covered by an inertial surface composed of a thin uniformly distributed matter of area density $\rho \varepsilon(0 \leqslant \varepsilon<1)$. We assume its motion to be under gravity only and small, and let the motion commence at time $t=0$ from a stage of rest; it is then irrotational and can be described by a velocity potential $\varphi(x, y, z ; t)$ for $t>0$.

Assuming linearized theory, $\varphi$ satisfies the Laplace's equation

$$
\nabla^{2} \varphi=0, \quad 0<y<h,
$$

except at a point of singularity, if any. As discussed in [3] $\varphi$ can be shown to satisfy the initial conditions

$$
\varphi-\varepsilon \partial \varphi / \partial y=0, \quad \partial / \partial t(\varphi-\varepsilon \partial \varphi / \partial y)=0 \quad \text { on } y=0 \text { at } t=0
$$

the linearized inertial surface condition

$$
\partial^{2} / \partial t^{2}(\varphi-\varepsilon \partial \varphi / \partial y)-g \partial \varphi / \partial y=0 \text { on } y=0,
$$

and the bottom condition

$$
\partial \varphi / \partial y=0 \text { on } y=h .
$$

It may be noted that for a time-harmonic motion of circular frequency $\sigma$, the inertial surface condition (2.3) becomes

$$
K \varphi+(1-K \varepsilon) \partial \varphi / \partial y=0 \text { on } y=0
$$


where $K=\sigma^{2} / g$. For $0 \leqslant K \varepsilon<1$, this is of the form

$$
K^{*} \varphi+\partial \varphi / \partial y=0 \text { on } y=0
$$

where

$$
K^{*}=K(1-K \varepsilon)^{-1},
$$

and (2.6) is thus merely a modification of the usual free-surface condition. However, as noted in [4], for $K \varepsilon \geqslant 1$ the condition (2.5) does not allow propagation of progressive waves.

Let $\bar{\varphi}(x, y, z ; p)$ denote the Laplace transform of $\varphi(x, y, z ; t)$ defined as

$$
\bar{\varphi}=\int_{0}^{\infty} \exp (-p t) \varphi d t \quad(p>0)
$$

then $\bar{\varphi}$ is the solution of the boundary-value problem described by

$$
\nabla^{2} \bar{\varphi}=0, \quad 0<y<h,
$$

except at a point of singularity,

$$
p^{2} \bar{\varphi}-\left(g+\varepsilon p^{2}\right) \partial \bar{\varphi} / \partial y=0 \text { on } y=0,
$$

and

$$
\partial \bar{\varphi} / \partial y=0 \text { on } y=h .
$$

$\bar{\varphi}$ can be obtained in a manner analogous to the corresponding time-harmonic problems in a liquid with a free surface. Laplace inversion will then give $\varphi$.

\section{Line singularities}

Let a line singularity be placed at the point $(X, Y)(Y>0)$ so that the potential denoted by $G(x, y ; X, Y ; t)$, say, describes the symmetric motion due to a submerged wave source where the motion is two-dimensional and the potential is a function of $x, y$ and $t$. Hence $G$ satisfies the initial value problem described by (2.1) to (2.4) and

$$
G \sim m(t) \ln \rho \quad \text { as } \rho=\left\{(x-X)^{2}+(y-Y)^{2}\right\}^{1 / 2} \rightarrow 0,
$$

where $m(t)$ is the time-dependent strength of the source. Then $\bar{G}(x, y ; X, Y ; p)$ satisfies the boundary-value problem described by (2.8) to (2.10) and

$$
\bar{G} \sim \bar{m}(p) \ln \rho \quad \text { as } \rho \rightarrow 0 .
$$

Thus, following Thorne [4], in the usual time-harmonic case $\bar{G}$ here can be represented by

$$
\begin{array}{r}
\bar{G}=\bar{m}\left[\ln \rho / \rho^{\prime}+\int_{0}^{\infty}\{A(k) \cosh k(h-y)\right. \\
+B(k) \sinh k y\} \cos k(x-X) d k],
\end{array}
$$


where $\rho^{\prime}=\left\{(x-X)^{2}+(y+Y)^{2}\right\}^{1 / 2}$ and $A$ and $B$ are unknown functions. Using the boundary conditions (2.9), (2.10), $A$ and $B$ can be determined uniquely. After rearrangement we obtain

$$
\begin{array}{r}
\bar{G}=\bar{m}\left[U(x, y)-2 \int_{0}^{\infty} \frac{\cosh k(h-Y) \cosh k(h-y)}{k D(k)}\right. \\
\left.\quad \times \frac{\cos k(x-X)}{\sinh k h} \frac{\mu^{2}}{\mu^{2}+p^{2}} d k\right]
\end{array}
$$

where

$$
\begin{gathered}
U=\ln \frac{\rho}{\rho^{\prime}}-2 \int_{0}^{\infty}\left\{\frac{\varepsilon \cosh k(h-Y) \cosh k(h-y)}{D(k)}\right. \\
\left.+\frac{\exp (-k h)}{k} \sinh k Y \sinh k y\right\} \frac{\cos k(x-X)}{\cosh k h} d k, \\
D(k)=\cosh k h+k \varepsilon \sinh k h,
\end{gathered}
$$

and

$$
\mu^{2}=g k \sinh k h / D(k) .
$$

Laplace inversion of (3.2) gives

$$
\begin{gathered}
G=m(t) U-2 \int_{0}^{\infty} \mu \frac{\cosh k(h-Y) \cosh k(h-y)}{k D(k)} \frac{\cos k(x-X)}{\sinh k h} \\
\times \int_{0}^{t} m(\tau) \sin \mu(t-\tau) d \tau d k
\end{gathered}
$$

Now we consider three particular forms of the source strength $m(t)$. When $m(t)=\delta(t)$, the corresponding potential of impulsive source strength is given by

$$
\begin{array}{r}
G^{\text {spec }}(t)=\delta(t) U-2 \int_{0}^{\infty} \mu \frac{\cosh k(h-Y) \cosh k(h-y)}{k D(k)} \\
\times \frac{\cos k(x-X)}{\sinh k h} \sin \mu t d k
\end{array}
$$

As

$$
t \rightarrow \infty, \quad G^{\text {spec }}(t) \rightarrow \lim _{p \rightarrow 0}\left(p \bar{G}^{\text {spec }}\right)=0 .
$$

This result can be explained from the fact that as the source acts only instantaneously at $t=0$, its effect will not be felt anywhere in the liquid region after a long lapse of time. 
The classical wave source of constant strength $m(t)=1$ has the potential given by

$$
\begin{aligned}
& G_{0}(t) \equiv G_{0}(x, y ; X, Y ; t) \\
& =U(x, y)-2 \int_{0}^{\infty} \frac{\cosh k(h-Y) \cosh k(h-y)}{D(k)} \frac{\cos k(x-X)}{\sinh k h} \frac{1-\cos \mu t}{k} d k .
\end{aligned}
$$

This coincides with the expression obtained by Finkelstein [1] for $\varepsilon=0$. This potential exists for finite time only. As $t \rightarrow \infty, G_{0}(t)$ does not possess a finite limit although grad $G_{0}$ has finite limit. In particular, as $t \rightarrow \infty$

$$
\begin{gathered}
\frac{\partial G_{0}(t)}{\partial x} \rightarrow \frac{\partial}{\partial x}\left(\ln \frac{\rho}{\rho^{\prime}}\right)+2 \int_{0}^{\infty}\left\{\frac{\cosh k(h-Y) \cosh k(h-y)}{\sinh k h}\right. \\
\left.-e^{-k h} \sinh k Y \sinh k y\right\} \frac{\sin k(x-X)}{\cosh k h} d k \\
\frac{\partial G_{0}(t)}{\partial y} \rightarrow \frac{\partial}{\partial y}\left(\ln \frac{\rho}{\rho^{\prime}}\right)+2 \int_{0}^{\infty}\left\{\frac{\cosh k(h-Y) \sinh k(h-y)}{\sinh k h}\right. \\
\left.-e^{-k h} \sinh k Y \cosh k y\right\} \frac{\cos k(x-X)}{\cosh k h} d k .
\end{gathered}
$$

This has an analogy in the electrodynamic theory, where the potential at any point due to charges uniformly distributed on an infinite straight line is infinite, although the force field remains finite.

When $m(t)=\sin \sigma t$, we obtain the potential due to a time-harmonic wave source as

$$
\begin{aligned}
G=\sin \sigma t U-2 \int_{0}^{\infty} & \mu \frac{\cosh k(h-y) \cosh k(h-Y)}{k D(k)} \\
& \times \frac{\cos k(x-X)}{\sinh k h} \frac{\mu \sin \sigma t-\sigma \sin \mu t}{\mu^{2}-\sigma^{2}} d k .
\end{aligned}
$$

Now to obtain the form of this potential as $t \rightarrow \infty$, following Rhodes-Robinson [3], we introduce a Cauchy principal value at $k=k_{0}^{*}$, which is the positive zero of $k \sinh k h-K^{*} \cosh k h$ when $0 \leqslant K \varepsilon<1$. Hence as $t \rightarrow \infty$, following [3], we obtain after simplification,

$$
\begin{aligned}
G \sim & \sin \sigma t\left[\ln \frac{\rho}{\rho^{\prime}}-2 f_{0}^{\infty}\left\{\frac{\cosh k(h-Y) \cosh k(h-y)}{k \sinh k h-K^{*} \cosh k h}\right.\right. \\
& \left.\left.+\frac{\exp (-k h)}{k} \sinh k y \sinh k Y\right\} \frac{\cos k(x-X)}{\cosh k h} d k\right] \\
+4 \pi & \frac{\cosh k_{0}^{*}(h-Y) \cosh k_{0}^{*}(h-y)}{\sinh 2 k_{0}^{*} h+2 k_{0}^{*} h} \cos k_{0}^{*}(x-X) \cos \sigma t .
\end{aligned}
$$


We next show that the wave source potential (3.11) satisfies the radiation condition. Following $\mathrm{Yu}$ and Ursell [6], we note that the integral

$$
\int_{0}^{\infty} \frac{\cosh k(h-Y) \cosh k(h-y) \exp (i k|x-X|)}{\left(k \sinh k h-K^{*} \cosh k h\right) \cosh k h} d k
$$

along the real $k$ axis (indented at $k=k_{0}^{*}$, where the integrand has a simple pole) is equal to the integral along $\arg k=\pi / 4$, and thus clearly tends to zero as $|x-X| \rightarrow \infty$. Thus

$$
\begin{aligned}
\int_{0}^{\infty} \frac{\cosh k(h-Y) \cosh k(h-y) \exp (i k|x-X|)}{k \sinh k h-K^{*} \cosh k h} d k-\left(\pi i \text { residue at } k=k_{0}^{*}\right) \\
\rightarrow 0 \text { as }|x-X| \rightarrow \infty .
\end{aligned}
$$

(The second term is the finite contribution from the indentation at the pole).

Taking real parts we find that

$$
\begin{aligned}
& f_{0}^{\infty} \frac{\cosh k(h-Y) \cosh k(h-y)}{k \sin k h-K^{*} \cosh k h} \frac{\cos k(x-X)}{\cosh k h} d k \\
& +2 \pi \frac{\cosh k_{0}^{*}(h-Y) \cosh k_{0}^{*}(h-y)}{\sinh 2 k_{0}^{*} h+2 k_{0}^{*} h} \sin k_{0}^{*}(|x-X|) \rightarrow 0 \text { as }|x-X| \rightarrow \infty,
\end{aligned}
$$

whence (3.11) takes the form

$$
4 \pi \frac{\cosh k_{0}^{*}(h-Y) \cosh k_{0}^{*}(h-y)}{\sinh 2 k_{0}^{*} h+2 k_{0}^{*} h} \cos \left(k_{0}^{*}|x-X|-\sigma t\right) \quad \text { as }|x-X| \rightarrow \infty \text {. }
$$

Thus the form of (3.11) represents an outgoing wave as $|x-X| \rightarrow \infty$.

When $K \varepsilon \geqslant 1$, there is no zero of $k(K \varepsilon-1) \sinh k h+K \cosh k h$ for $k>0$. Then by the Riemann-Lebesgue lemma the integral involving sin $\sigma$ in (3.10) is wholly transient and after simplification we obtain

$$
\begin{array}{r}
G-\sin \sigma t\left[\ln \frac{\rho}{\rho^{\prime}}-2 \int_{0}^{\infty}\left\{\frac{\cosh k(h-Y) \cosh k(h-y)}{k \sinh k h+K(K \varepsilon-1)^{-1} \cosh k h}\right.\right. \\
\left.\left.+\frac{\exp (-k h)}{k} \sinh k Y \sinh k y\right\} \frac{\cos k(x-X)}{\cosh k h} d k\right] .
\end{array}
$$

In this case there exists no outgoing wave as $|x-X| \rightarrow \infty$.

Making $h \rightarrow \infty$ all the results obtained here reduce to the corresponding results for infinite depth of liquid obtained in [3].

In the case of variable multipoles

$$
G \sim m(t) \cos n \theta / \rho^{n} \quad \text { as } \rho \rightarrow 0, n=1,2, \ldots
$$

where $x-X=\rho \sin \theta, y-Y=\rho \cos \theta$. But since

$$
\cos n \theta / \rho^{n}=-1 /(n-1) ! \partial^{n} \partial Y^{n}(\ln \rho),
$$


this potential function can be derived from the line source solution by differentiation of (3.6) with respect to $Y$. The final result is

$$
\begin{aligned}
G= & m(t)\left[\frac{\cos n \theta}{\rho^{n}}+\frac{1}{(n-1) !} \int_{0}^{\infty} \frac{k^{n-1}}{D(k)}[\exp \{-k(h-Y)\}\right. \\
& \times(k \varepsilon \cosh k y+\sinh k y) \\
& \left.\left.+(-1)^{n}(k \varepsilon-1) \exp (-k Y) \cosh k(h-y)\right] \cos k(x-X) d k\right] \\
+ & \frac{1}{(n-1) !} \int_{0}^{\infty} \frac{\mu k^{n-1}}{D(k)}\left[\exp \{-k(h-Y)\}+(-1)^{n} \exp \{k(h-Y)\}\right] \\
& \times \cosh k(h-y) \frac{\cos k(x-X)}{\sinh k h} \int_{0}^{t} m(\tau) \sin \mu(t-\tau) d \tau d k .
\end{aligned}
$$

\section{Variable point sources (multipoles) submerged in liquid of finite depth}

Let a point source of variable strength $m(t)$ be present at the point $(X, Y, 0)$ where $Y>0$. We consider only those sources for which the $y$-axis is an axis of symmetry. Let $\rho$ denote the distance from the $y$-axis and $R$ the distance from the source point. Let us define $\Psi$ such that

$$
\rho=R \sin \Psi, \quad y-Y=R \cos \Psi .
$$

Then in this case the potential $\varphi$ satisfies the three-dimensional Laplace's equation in the liquid region except at $(X, Y, 0)$, the initial conditions (2.2), the inertial surface condition (2.3), the bottom condition (2.4), and

$$
\varphi \sim m(t) P_{n}(\cos \Psi) / R^{n+1} \quad \text { as } R \rightarrow 0,
$$

where $P_{n}(z)$ is the Legendre polynomial of degree $n$. The final form of $\varphi$ is given by

$$
\begin{aligned}
\varphi= & m(t) V(x, y, z) \\
& +\frac{1}{n !} \int_{0}^{\infty} \mu k^{n} \frac{E(k)}{D(k)} \cosh k(h-y) \frac{J_{0}(k \rho)}{\sinh k h} \int_{0}^{t} m(\tau) \sin \mu(t-\tau) d \tau d k,
\end{aligned}
$$


where

$$
\begin{gathered}
V=\frac{P_{n}(\cos \Psi)}{R^{n+1}} \frac{1}{n !} \int_{0}^{\infty} \frac{k^{n}}{D(k)}[\exp \{-k(h-Y)\}(\varepsilon K \cosh k y+\sinh k y) \\
\left.+(-1)^{n}(\varepsilon k-1) \exp (-k Y) \cosh k(h-y)\right] J_{0}(k \rho) d k \\
E(k)=\exp \{-k(h-Y)\}+(-1)^{n} \exp \{k(h-Y)\}
\end{gathered}
$$

and $J_{0}(k \rho)$ is the Bessel function of first kind and $D$ and $\mu$ are given in $\S 3$.

\section{Conclusion}

Potential functions due to different types of singularities with time-dependent strengths submerged in a liquid of finite depth with an inertial surface are obtained. Making $h \rightarrow \infty$ the corresponding results for infinite depth of the liquid can be derived. Putting $\varepsilon=0$, the results reduce to those for a liquid with a free surface. As mentioned in [3], these results can be extended to include the effect of surface tension at the inertial surface, to the case of two superposed liquids of either infinite or finite constant depth and height of the liquids that are separated by an inertial surface.

\section{Acknowledgement}

One of the authors $(K K)$ thanks the Calcutta University for the award of a U.G.C. Research Fellowship. We also thank the referees and the Associate Editor for their comments and suggestions in improving the manuscript considerably.

\section{References}

[1] A. B. Finkelstein, "The initial-value problem for transient water waves", Comm. Pure Appl. Math. 10 (1957), 511-522.

[2] P. F. Rhodes-Robinson, "Fundamental singularities in the theory of water waves with surface tension", Bull A ustral. Math. Soc, 2 (1970), 317-333.

[3] P. F. Rhodes-Robinson, "On the generation of water waves at an inertial surface", $J$. Austral. Math. Soc. Ser. B 25 (1984), 366-383.

[4] P. F. Rhodes-Robinson, "Note on the effect of surface tension on water waves at an inertial surface", J. Fluid Mech. 125 (1982), 375-377.

[5] R. C. Thorne, "Multipole expansions in the theory of surface waves", Proc. Camb. Phil. Soc. 49 (1953), 707-716.

[6] Y. S. Yu and F. Ursell, "Surface waves generated by an oscillating circular cylinder on water of finite depth: theory and experiment", J. Fluid Mech., 11 (1961), 529-551. 